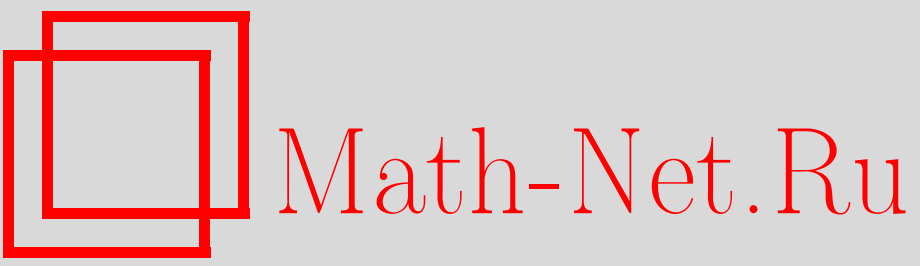

А. М. Переломов, Замечание о матричной функции Эйри, ТМФ, 2000, том 123, номер 2, 308-310

DOI: https://doi.org/10.4213/tmf605

Использование Общероссийского математического портала Math-Net.Ru подразумевает, что вы прочитали и согласны с пользовательским соглашением

http://www.mathnet.ru/rus/agreement

Параметры загрузки:

IP: 34.239 .49 .27

26 апреля 2023 г., $17: 55: 27$ 


\section{ЗАМЕЧАНИЕ О МАТРИЧНОЙ ФУНКЦИИ ЭЙРИ}

Получено новое интегральное представление матричной функции Эйри.

\section{Памяти М. В. Савельева}

Хорошо известная гипотеза Виттена [1] о том, что логарифм статистической суммы обшей одноматричной модели является производящей функцией индексов пересечения на пространстве модулей алгебраических кривых, была доказана Концевичем [2, 3], который свел эту задачу к вычислению матричной функции Эйри. Многие аспекты этой проблемы обсуждались в работах [4-6].

Матричная функция Эйри определяется с помощью интегрального представления. В данной статье дано еще одно интегральное представление этой функции.

Следуя работе [3], определим матричную функцию Эйри на пространстве $\mathcal{H}_{N}$ эрмитовых $(N \times N)$-матриц как интеграл

$$
A(X)=\int \exp \left\{i\left(\frac{1}{3} \operatorname{tr}\left(Y^{3}\right)-\operatorname{tr}(X Y)\right)\right\} d Y
$$

по пространству $\mathcal{H}_{N}$, где $d Y$ есть $U(N)$-инвариантная мера на этом пространстве, $X, Y \in \mathcal{H}_{N}$.

Функция $A(X)$ удовлетворяет уравнению

$$
\Delta A(X)+(\operatorname{tr} X) A(X)=0
$$

где $\Delta$ - оператор Лапласа.

Напомним, что классическая функция Эйри

$$
\operatorname{Ai}(x)=\int_{-\infty}^{+\infty} \exp \left(i\left(\frac{y^{3}}{3}-x y\right)\right) d y
$$

есть единственное (с точностью до множителя) ограниченное на вешественной оси решение дифференциального уравнения

$$
\operatorname{Ai}^{\prime \prime}(x)+x \operatorname{Ai}(x)=0 .
$$

${ }^{*}$ Институт математики им. Макса Планка, Бонн, Германия; Институт теоретической и экспериментальной физики, Москва, Россия. E-mail:perelomo@posta.unizar.es 
Из определения (1) следует, что зависимость функции $A(X)$ от переменных $\widetilde{X}$ и $\xi=$ $N^{-1} \operatorname{tr} X(X=\widetilde{X}+\xi I, \operatorname{tr} \widetilde{X}=0)$ можно разделить. Действительно,

$$
\begin{gathered}
X=\tilde{X}+\xi I, \quad Y=\tilde{Y}+\eta I, \quad \operatorname{tr} \tilde{X}=\operatorname{tr} \tilde{Y}=0, \\
\operatorname{tr}\left(Y^{3}\right)=\operatorname{tr}\left(\tilde{Y}^{3}\right)+3 \eta \operatorname{tr}\left(\widetilde{Y}^{2}\right)+N \eta^{3} \\
\operatorname{tr}(X Y)=\operatorname{tr}(\tilde{X} \widetilde{Y})+N \xi \eta .
\end{gathered}
$$

ТЕОРема 1. Для матричной функиии Эйри (1) справедливо интегральное представление

$$
\begin{aligned}
A(X) & =A(\tilde{X}, \xi)=A(Q, \xi)= \\
& =C N^{-\frac{1}{3}} \int \exp \left\{\frac{i}{3} \operatorname{tr}\left(P^{3}\right)\right\} \operatorname{Ai}\left(N^{-\frac{1}{3}}\left(N \xi-\operatorname{tr}\left(P^{2}\right)\right)\right) \Phi(Q \mid P) d \mu(P)
\end{aligned}
$$

Здесь $Q$ и $P$ - диагональные матрицы такие, что $\tilde{X}=U Q U^{-1}, \tilde{Y}=U_{1} P U_{1}^{-1}, U$ и $U_{1}$ - унитарнье матрицы, $\Phi(Q \mid P)$ - зональная сферическая функция,

$$
\begin{gathered}
\Phi(Q \mid P)=\frac{\operatorname{det}\left\{\exp \left(i\left(q_{j} p_{k}\right)\right)\right\}}{\operatorname{det}\left\{\left(q_{j} p_{k}\right)\right\}}, \\
Q=\operatorname{diag}\left(q_{1}, \ldots, q_{N}\right), \quad P=\operatorname{diag}\left(p_{1}, \ldots, p_{N}\right), \quad d \mu(P)=(V(P))^{2} d P,
\end{gathered}
$$

$C=$ const, $V(P)$ - определитель Вандермонда,

$$
V(P)=\prod_{j<k}\left(p_{j}-p_{k}\right)=\operatorname{det}\left(p_{j}^{k-1}\right) .
$$

ДокАЗАТЕЛьство. Доказательство следует из формулы Хариш-Чандры.

ТеОРема 2 [7]. Пусть $\Phi$ - функиия на пространстве эрмитовых $(N \times N)$-матрии, инвариантная относительно сопряжения. Тогда для любой диагональной әрмитовой матрици $Q$ справедливо равенство

$$
\begin{aligned}
& \int \Phi(Y) \exp (-i \operatorname{tr}(Q Y)) d Y= \\
& \quad=(-2 \pi i)^{\frac{N(N-1)}{2}}(V(Q))^{-1} \int \Phi(P) \exp (-i \operatorname{tr}(Q P))(V(P))^{2} d P
\end{aligned}
$$

где $Y=U P U^{-1}$ и интегрирование в последнем интеграле ведется по пространству диагональных эрмитовых матрии $P$.

Полученное интегральное представление матричной функции Эйри, в котором зависимость от переменной $\xi$ содержится только в стандартной одномерной функции Эйри, может быть полезно при исследовании свойств матричной функции Эйри. 
В случае $N=2$, когда $\operatorname{tr}\left(P^{3}\right)=0$, интегральное представление (5) принимает более простой вид

$$
A(X) \equiv A(\xi, r)=\int \exp \left\{\frac{2 i}{3}\left(\eta^{3}+3 \eta p^{2}-3 \xi \eta\right)\right\} \Phi(r \mid p) p^{2} d p d \eta
$$

где

$$
\Phi(r \mid p)=\frac{\sin p r}{p r}
$$

есть соответствуюшая зональная сферическая функция. Интегрирование по $\eta$ дает

$$
A(\xi, r)=C \int \operatorname{Ai}\left(\left(\frac{3}{2}\right)^{\frac{1}{3}}\left(\xi-p^{2}\right)\right) \Phi(r \mid p) p^{2} d p, \quad C=2\left(\frac{3}{2}\right)^{\frac{1}{3}}
$$

Если в формуле (10) взять интеграл по $p$, получим другое интегральное представление

$$
A(\xi, r)=C_{1} \int \exp \left\{\frac{2 i}{3}\left(\tau^{3}-3 \xi \tau\right)\right\} G(r, \tau) d \tau, \quad C_{1}=\text { const }
$$

где

$$
G(r, \tau)=\tau^{-\frac{3}{2}} \exp \left\{i \tau^{-1} r^{2}\right\}
$$

- функция Грина свободного уравнения Шредингера в трехмерном пространстве.

Благодарности. Эта работа была выполнена во время моего пребывания в Институте математики им. Макса Планка в Бонне. Мне приятно поблагодарить сотрудников этого института за гостеприимство.

\section{Список литературы}

[1] E. Witten. J. Diff. Geom., Suppl. 1991. V. 1. P. 243-310.

[2] М. Концевич. Функц. анализ и его прилож. 1991. Т. 25. № 2. С. 50-57.

[3] M. Kontsevich. Commun. Math. Phys. 1992. V. 147. P. 1-23.

[4] E. Witten. On the Kontsevich model and other models of two-dimensional gravity. Princeton, N.Y.: Princeton Univ. Press, 1991.

[5] E. Witten. Nucl. Phys. B. 1992. V. 371. P. 191-245.

[6] C. Itzykson, J.-B. Zuber. Int. J. Mod. Phys. A. 1992. V. 7. P. 5661-5705.

[7] Harish-Chandra. Am. J. Math. 1957. V. 79. P. 87-120. 